\title{
Čech Cocycles for Characteristic Classes
}

\author{
J.-L. Brylinski ${ }^{1, \star}$, D.A. McLaughlin ${ }^{2, \star \star}$ \\ ${ }^{1}$ Mathematics Department, Pennsylvania State University, University Park, PA 16802, USA \\ 2 Mathematics Department, Fine Hall, Princeton University, Princeton, NJ 08544-1000, USA
}

Received: 19 May 1995

\begin{abstract}
We give general formulae for explicit Čech cocycles representing characteristic classes of real and complex vector bundles, as well as for cocycles representing Chern-Simons classes of bundles with arbitrary connections. Our formulae involve integrating differential forms over moving simplices inside homogeneous spaces. An important feature of our cocycles is that they take integer values (as opposed to real or rational values). We find in particular a formula for the instanton number of a connection over a closed four-manifold with arbitrary structure group. For flat connections, our formulae recover and generalize those of Cheeger and Simons. The methods of this paper apply also to the purely geometric construction of the Quillen line bundle with its metric.
\end{abstract}

A vector bundle $E \rightarrow M$ has characteristic classes (Chern, Pontryagin and Euler classes) in integral cohomology groups $H^{p}(M, \mathbb{Z})$. The Chern-Weil theory gives differential forms which represent the corresponding classes in the real cohomology groups $H^{p}(M, \mathbb{R})$. Gelfand posed the problem of finding a combinatorial formula for integer-valued singular cocycles representing the Pontryagin classes. This is considerably more difficult than finding a real-valued cocycle, which can easily be done using a partition of unity [5].

An explicit formula for a singular cocycle representing 24 times the first Pontryagin class $p_{1}(M)$ of a smooth manifold $M$ was found by I. Gelfand, Gabrielov and Losik [16] and by MacPherson [20]. This formula, which involves the dilogarithm function, has had considerable influence in algebraic topology and in algebraic K-theory. More recently, Gelfand and MacPherson [17] gave a formula for a $\mathrm{ra}$ tional simplicial cocycle representing any Pontryagin class of a smooth polyhedral manifold.

In this paper we work with Čech cohomology instead of singular cohomology. We give a direct and explicit construction of all the integer-valued Čech cocycles

$\star$ The first author was supported in part by N.S.F. grant DMS-9203517.

$\star \star$ The second author was supported in part by N.S.F. grant DMS-9310433. 
which represent a given characteristic class. We adopt the following point of view. A vector bundle may be completely described by a set $\left\{g_{i j}\right\}$ of transition cocycles, relative to some suitable open covering of $M$. We construct the integer-valued Čech cocycles representing a characteristic class directly from the data $g_{i j}$. We obtain Čech cocycles representing the Pontryagin classes $p_{k} \in H^{4 k}(M, \mathbb{Z})$ and the Euler class $e \in H^{n}(M, \mathbb{Z})$ of a real vector bundle of rank $n$, and the Chern classes $c_{k} \in H^{2 k}(M, \mathbb{Z})$ of a complex vector bundle (see Theorems 2,3 and 4). The only denominators which are needed are some powers of 2 in the case of $p_{k}$ for $k>1$. These formulae generalize the formula for $p_{1}$ announced in [6].

Our formula may also be viewed as the completion of a project of Chern and Simons, who explain in the introduction to [12] that their secondary characteristic classes "grew out of an attempt to derive a purely combinatorial formula for the first Pontryagin number of a 4-manifold." The "boundary term" which Chern and Simons say "did not yield to simple combinatorial analysis" is incorporated in the formulae given in this paper.

All the characteristic classes above may be defined by transgression in an associated bundle, with fiber a Stiefel manifold [4]. We realize this transgression geometrically, by constructing "moving cycles," which are moving families of singular cycles on the Stiefel manifold, parametrized by various open subsets of $M$. This construction is contained in the first section, along with the statement of Theorems 2, 3 and 4.

The proof of these theorems is given in Sects. 2 and 3. In fact, much more is shown. In Sect. 2, by choosing a connection on $E \rightarrow M$, we exhibit lifts of $p_{k}, e$ and $c_{k}$ to characteristic classes in Cheeger-Simons cohomology-or in smooth Deligne cohomology (see Theorem 5). In Sect. 3, we show that these lifts agree with the classes of Chern-Cheeger-Simons [11,12]. We also recover formulae of Cheeger-Simons [11] and of Dupont [13,14] for flat bundles and we generalize them to the case where the $g_{i j}$ are not in general position. In Sect. 4, we look at some interesting special cases of our formulae. For the second Chern class of a principal $S U(n)$-bundle, the formula simplifies considerably and involves computing the integral of the Chern-Simons 3-form $v$ over a tetrahedron in $S U(n)$ whose vertices are the transition functions. The corresponding Chern-Cheeger-Simons class can then be written explicitly in terms of $v$. For a four-dimensional manifold, this gives a formula for the topological charge or instanton number. In the case of $S U(2)$, this was essentially known to Laursen, Schierholz and Wiese [19]. Thus we obtain an extension of their formulae for arbitrary classical compact groups. For the first Pontryagin class, we also obtain an explicit formula, but the tetrahedron has to be altered slightly due to the 2-torsion in $\pi_{1} S O(n)$.

Finally, A. Goncharov [18] has constructed Chern classes in grassmannian cohomology and he has also constructed explicitly $c_{2}$ and $c_{3}$ in motivic cohomology. This in particular implies formulae for these classes in Deligne cohomology and in ordinary cohomology.

We would like to thank Sasha Beilinson, Pierre Deligne, Israel Gelfand, Sasha Goncharov, David Kazhdan and Bob MacPherson for useful discussions. 


\section{The Formulae}

In this section, we will write down an explicit $\mathbb{Z}$-valued Čech cocycle representing each of the following characteristic classes:

- the Euler class $e$ and the Pontryagin classes $p_{k}$ of a principal $S O(n)$-bundle;

- the Chern classes $c_{k}$ of a principal $U(n)$-bundle

Let $G$ be a Lie group, let $M$ be a compact manifold and let $p: P \rightarrow M$ be a principal bundle with structure group $G$. By convention, we write the $G$-action on $P$ as a right action, and use the notation $x \cdot g$ for the action of $g \in G$ on $x \in P$. We choose a good open covering $\mathscr{U}=\left(U_{i}\right)$ of $M$, i.e. we assume that all intersections $U_{i_{1} \cdots i_{k}}:=U_{i_{1}} \cap \cdots \cap U_{i_{k}}$ are contractible or empty (see [22]). Let $s_{i}: U_{i} \rightarrow P$ be a family of smooth local sections of $p$. Then the transition cocycles $g_{i j}$ are the smooth functions $g_{i j}: U_{i j} \rightarrow G$ defined by the formula $s_{j}=s_{i} \cdot g_{i j}$.

Cycle associated to $p_{k}$. We start from the data of a principal $S O(n)$-bundle $p$ : $P \rightarrow M$, where $n$ is odd. We introduce the real Stiefel manifold $V_{n, q}=S O(n) / S O(q)$; for $g \in S O(n)$, we denote by $\bar{g}$ the image of $g$ in the coset space $S O(n) / S O(q)$. The image $\overline{1}$ of 1 in $V_{n, q}$ is the base point of $V_{n, q}$. The reduced homology $\tilde{H}_{i}\left(V_{n, 2 k-1}, \mathbb{Z}\right)$ is well known [3]. We have:

$$
\tilde{H}_{i}\left(V_{n, 2 k-1}, \mathbb{Z}\right)=\left\{\begin{array}{l}
\mathbb{Z} / 2 \text { for } i \text { odd, } 2 k-1 \leqq i \leqq 4 k-2 \\
\mathbb{Z} \oplus(2-\text { primary group }) \text { for } i=4 k-1 \\
0 \text { if } i \leqq 4 k-3, i \text { even or } i \leqq 2 k-2
\end{array}\right.
$$

We will work with the groups $C_{j}\left(i_{0}, \ldots, i_{m}\right)$ of smooth singular $j$-chains for the manifold of smooth maps from $U_{i_{0} \cdots i_{m}}$ to $V_{n, 2 k-1}$. Note that such a chain may be viewed as a linear combination of smooth mappings $\sigma: U_{i_{0} \cdots i_{m}} \times \Delta^{j} \rightarrow$ $V_{n, 2 k-1}$, where $\Delta^{j}$ is the standard $j$-simplex. We have the usual boundary map $\partial: C_{j}\left(i_{0}, \ldots, i_{m}\right) \rightarrow C_{j-1}\left(i_{0}, \ldots, i_{m}\right)$.

Lemma 1. There exists a family of elements $\sigma_{i_{0} \cdots i_{j}}^{j}$ of $C_{j}\left(i_{0}, \ldots, i_{j}\right)$, where $j$ ranges over $\{0,1, \ldots, 4 k-1\}$, and $i_{0}, \ldots, i_{j}$ range over $j+1$-tuples of elements of $I$, which satisfies

$$
\sigma_{i}^{0}(y)=\overline{1} \quad \text { for } i \in I, y \in U_{i},
$$

and

$$
\partial \sigma_{i_{0} \cdots i_{j}}^{j}=a_{j-1} \cdot\left[g_{i_{0} i_{1}} \cdot \sigma_{i_{1} \cdots i_{j}}^{j-1}+\sum_{l=1}^{j}(-1)^{l} \sigma_{i_{0} \cdots \hat{l}_{l} \cdots i_{j}}^{j-1}\right] \quad \text { for } j \geqq 1
$$

We explain in more detail the meaning of (2). In the right-hand side of (2), we let $g_{i_{0} i_{1}}$ operate on smooth maps from $U_{i_{0} \cdots i_{j}} \times \Delta^{j} \rightarrow V_{n, 2 k-1}$ via the action of $S O(n)$ on $V_{n, 2 k-1}$, and this is extended linearly to $j$-chains. We denote by the same letter a chain like $\sigma_{i_{1} \cdots i_{j}}^{j-1}$ and its restriction to $\operatorname{Map}\left(U_{i_{0} \cdots i_{j}}, V_{n, 2 k-1}\right)$. Finally, the number $a_{j}$ is the cardinality of the reduced homology group $\tilde{H}_{j}\left(V_{n, 2 k-1}, \mathbb{Z}\right)$.

Proof. The chains $\sigma_{i_{0} \cdots i_{j}}^{j}$ are constructed by induction on $j$. For $j=0$, we define $\sigma_{i}^{0}(y)=\overline{1}$ so that $(1)$ is verified. Given $j \geqq 1$ and $i_{0}, \ldots, i_{j}$, we may assume 
that $U_{i_{0} \cdots i_{j}}$ is not empty, hence contractible. The singular chain $g_{i_{0} i_{1}} \cdot \sigma_{i_{1} \cdots i_{j}}^{j-1}+$ $\sum_{l=1}^{j}(-1)^{l} \sigma_{i_{0} \cdots \hat{i}_{l} \cdots i_{j}}^{j-1}$ is seen to be a smooth singular cycle in the manifold $\operatorname{Map}\left(U_{i_{0} \cdots i_{j}}, V_{n, 2 k-1}\right)$, which is homotopy equivalent to $V_{n, 2 k-1}$. This cycle, when multiplied by $a_{j}$, becomes the boundary of some chain $\sigma_{i_{0} \cdots i_{j}}^{j}$.

We now define the smooth singular $4 k-1$ cycle $X_{i_{0} \cdots i_{4 k}}$ in $\operatorname{Map}\left(U_{i_{0} \cdots i_{4 k}}, V_{n, 2 k-1}\right)$ by the equality

$$
X_{i_{0} \cdots i_{4 k}}=g_{i_{0} i_{1}} \cdot \sigma_{i_{1} \cdots i_{4 k}}^{4 k-1}+\sum_{l=1}^{4 k}(-1)^{l} \sigma_{i_{0} \cdots \hat{i}_{l} \cdots i_{4 k}}^{4 k-1} .
$$

We may think of $X_{i_{0} \cdots i_{4 k}}$ as a smooth family of cycles $X_{l_{0} \cdots i_{4 k}}(y)$ on $V_{n, 2 k-1}$, parametrized by $y \in U_{i_{0} \cdots i_{4 k}}$.

Let $\Omega$ be the closed $S O(n)$-invariant form generating $H^{4 k-1}\left(V_{n, 2 k-1} ; \mathbb{Z}\right)$ modulo torsion. The Pontryagin class $p_{k}$ of the bundle $p: P \rightarrow M$ is defined to be the transgression of $2 \cdot \Omega$ in the associated bundle $\pi: P \times_{S O(n)} V_{n, 2 k-1} \rightarrow M$ [4]. For $y \in U_{i_{0} \cdots i_{4 k}}$, we let $\int_{X_{i_{0} \cdots i_{4 k}}(y)} \Omega$ be the number obtained by integration of $\Omega$ on the cycle $X_{i_{0} \cdots i_{4 k}}(y)$.

Theorem 2. (I) For each $i_{0}, \ldots, i_{4 k} \in I$, the function $y \mapsto \int_{X_{i_{0} \cdots i_{n}}(y)} \Omega$ is a constant function from $U_{i_{0} \cdots i_{4 k}}$ to $\mathbb{Z}$, denoted by $\int_{X_{L_{0} \cdots l_{4 k}}} \Omega$.

(II) The Čech $4 k$-cochain $\int_{X_{i_{0}} \cdots i_{4 k}} \Omega$ is a $\mathbb{Z}$-valued cocycle, whose cohomology class is equal to $-2^{k-1} \cdot p_{k}$.

(III) Any $\mathbb{Z}$-valued Čech cocycle which represents $-2^{k-1} \cdot p_{k}$ is obtained by this procedure, for a suitable choice of $\sigma_{i_{0} \cdots i_{j}}^{j}$.

We will now give the similar constructions which are appropriate for the Euler class and for the Chern classes.

Cycle associated to $e$. Again let $p: P \rightarrow M$ be an $S O(n)$-bundle, but we now assume $n$ even. As in Lemma 1, we can construct a family $\gamma_{i_{0} \cdots i_{j}}^{j}$ of smooth singular $j$-chains in $\operatorname{Map}\left(U_{i_{0} \cdots i_{j}}, S^{n-1}\right)$, defined for $j \leqq n-1$, satisfying the analog of (1) as well as

$$
\partial \gamma_{i_{0} \cdots i_{j}}^{j}=g_{i_{0} i_{1}} \cdot \gamma_{l_{1} \cdots i_{j}}^{j-1}+\sum_{l=1}^{j}(-1)^{l} \gamma_{i_{0} \cdots i_{l} \cdots i_{j}}^{j-1}
$$

Then we define $Y_{i_{0} \cdots i_{n}}$ to be the smooth singular $n$-cycle of $\operatorname{Map}\left(U_{i_{0} \cdots i_{n}}, S^{n-1}\right)$ given by

$$
Y_{i_{0} \cdots l_{n}}=g_{i_{0} i_{1}} \cdot \gamma_{i_{1} \cdots i_{n}}^{n-1}+\sum_{l=1}^{n}(-1)^{l} \gamma_{i_{0} \cdots i_{l} \cdots i_{n}}^{n-1}
$$

Let $\Lambda$ be the $S O(n)$-invariant form generating $H^{n-1}\left(S^{n-1} ; \mathbb{Z}\right)$. The Euler class $e$ may be defined as the transgression of $\Lambda$ in the sphere bundle associated to $P \rightarrow M$.

Theorem 3. (I) For each $i_{0}, \ldots, i_{n} \in I$, the function $y \mapsto \int_{Y_{l_{0} \cdots l_{n}(y)}} \Lambda$ is a constant function from $U_{i_{0} \cdots i_{n}}$ to $\mathbb{Z}$, denoted by $\int_{Y_{l_{0} \cdots i_{n}}} \Lambda$. 
(II) The Čech $n$-cochain $\int_{Y_{l_{0} \cdots l_{n}}} \Lambda$ is a $\mathbb{Z}$-valued cocycle, whose cohomology class is equal to $(-1)^{\frac{n}{2}+1} \cdot e$.

(III) Any $\mathbb{Z}$-valued $\breve{C}$ ech cocycle which represents $(-1)^{\frac{n}{2}+1} \cdot e$ is obtained by this procedure, for a suitable choice of $\gamma_{i_{0} \cdots i_{j}}^{j}$.

Cycle associated to $c_{k}$. Now let $p: P \rightarrow M$ be a principal $U(n)$-bundle. We introduce the complex Stiefel manifold $W_{n, q}=U(n) / U(q)$. The homology of $W_{n, q}$ is zero in degrees $\leqq 2 q$ and is equal to $\mathbb{Z}$ in degree $2 q+1$ [4]. We construct a family $\tau_{i_{0} \cdots i_{j}}^{j}$ of smooth $j$-chains in $\operatorname{Map}\left(U_{i_{0} \cdots i_{j}}, W_{n, k-1}\right)$ which satisfy the analog of (1) as well as

$$
\partial \tau_{i_{0} \cdots i_{J}}^{j}=g_{i_{0} i_{1}} \cdot \tau_{i_{1} \cdots i_{J}}^{j-1}+\sum_{l=1}^{j}(-1)^{l} \tau_{i_{0} \cdots \hat{i}_{l} \cdots i_{J}}^{j-1} .
$$

We let $Z_{i_{0} \cdots i_{2 k}}$ be the smooth $2 k-1$-cycle in $\operatorname{Map}\left(U_{i_{0} \cdots i_{2 k}}, W_{n, k-1}\right)$ defined by

$$
Z_{i_{0} \cdots i_{2 k}}=g_{i_{0} i_{1}} \cdot \tau_{i_{1} \cdots i_{2 k}}^{2 k-1}+\sum_{l=1}^{2 k}(-1)^{l} \tau_{i_{0} \cdots \hat{i}_{l} \cdots i_{2 k}}^{2 k-1} .
$$

Let $\Theta$ be the $S U(n)$-invariant form generating $H^{2 k-1}\left(W_{n, k-1} ; \mathbb{Z}\right)$. The Chern class $c_{k}$ may be defined as the transgression of $\Theta$ in the associated bundle $P \times S U(n)$ $W_{n, k-1} \rightarrow M[3]$.

Theorem 4. (I) For each $i_{0}, \ldots, i_{2 k} \in I$, the function $y \mapsto \int_{Z_{i_{0} \cdots l_{2 k}}(y)} \Theta$ is a constant function from $U_{i_{0} \cdots i_{2 k}}$ to $\mathbb{Z}$, denoted by $\int_{Z_{i_{0} \cdots i_{2 k}}} \Theta$.

(II) The Čech $2 k$-cochain $\int_{Z_{l_{0} \cdots l_{2 k}}(y)} \Theta$ is a $\mathbb{Z}$-valued cocycle, whose cohomology class is equal to $(-1)^{k-1} \cdot c_{k}$.

(III) Any $\mathbb{Z}$-valued $\breve{C}$ ech cocycle which represents $(-1)^{k-1} \cdot c_{k}$ is obtained by this procedure, for a suitable choice of the $\tau_{i_{0} \cdots i_{j}}^{j}$.

\section{Remarks}

1. Volumes of simplices also appear in [11]. These are geodesic simplices defined on the sphere bundle associated to a flat bundle.

2. There is a combinatorial formula for the integral first Pontryagin class of a simplicial manifold $[17,20]$, but we do not know whether our approach sheds any new light on this. We note the combinatorial formula of [10] for the signature of a closed oriented manifold.

3. A geometric proof of the formula for $p_{1}$ was given in [8]. This proof involved the notion of a 2-gerbe. It is expected that the general result can be proved using $n$-gerbes, although this has not yet been formulated precisely.

\section{The Proof}

We need only give the proof of Theorem 2, the other proofs being similar (and in fact slightly simpler). Let $\beta_{i_{0} \cdots i_{4 k}}=\int_{X_{l_{0} \cdots l_{4 k}}} \Omega$ denote the Čech cocycle which is 
our candidate for $-2^{k-1} p_{k}$. Choose a connection on $p: P \rightarrow M$, and let $\alpha$ be the $4 k$-form which is the Chern-Weil representative of $p_{k}$. Let $S=P \times_{S O(n)} V_{n, 2 k-1}, \pi$ : $S \rightarrow M$ the projection. The pullback of $\alpha$ to $S$ is exact, so there is a ( $4 k-1)$-form $\tilde{\Omega}$ on $S$ with $d \tilde{\Omega}=\frac{\pi^{*} \alpha}{2}$. In fact, for a given connection, there is a natural choice of a form $\tilde{\Omega}$ with this property $[10,11]$. The restriction of $\tilde{\Omega}$ to the fiber is $\Omega$.

We denote by $\bar{s}_{l}$ the trivialization $\bar{s}_{i}: U_{i} \times V_{n, 2 k-1} \stackrel{\sim}{\rightarrow} \pi^{-1}\left(U_{i}\right)$ induced by the section $s_{i}$ of $P$ over $U_{i}$. Define

$$
f_{i_{0} \cdots i_{4 k-1}}(y)=\exp \left(2 \pi i \cdot \int_{\substack{\sigma_{0} \cdots i_{4 k-1} \\ i_{0} \cdots}} \Omega\right), \quad \text { for } y \in U_{i_{0} \cdots i_{4 k-1}} .
$$

This is a degree $(4 k-1)$ cocycle with values in the sheaf $\underline{\mathbb{I}}$ of smooth circlevalued functions. Its image under the boundary map in the exponential exact sequence is $2 \pi i \cdot \beta_{i_{0} \cdots i_{4 k}}$. Using the trivialization $\bar{s}_{i}$, we associate to any smooth map $\sigma: U_{i_{0} \cdots i_{l}} \times \Delta^{l} \rightarrow V_{n, 2 k-1}$ a smooth map $\bar{\sigma}: U_{i_{0} \cdots i_{l}} \times \Delta^{l} \rightarrow S$ such that $\pi \circ \bar{\sigma}$ is the first projection $U_{i_{0} \cdots i_{j}} \times \Delta^{l} \rightarrow M$. We extend this linearly to smooth singular chains. Thus to $\sigma_{i_{0} \cdots i_{l}}^{l}$ we associate a smooth singular $l$-chain $\tilde{\sigma}_{i_{0} \cdots i_{l}}^{l}$ in $\operatorname{Map}\left(U_{i_{0} \cdots i_{l}}, S\right)$. We have the following expression for the boundary of these chains:

$$
\partial \tilde{\sigma}_{i_{0} \cdots i_{j}}^{j}=a_{j}\left[\tilde{\sigma}_{i_{1} \cdots i_{j}}^{j-1}+\sum_{l=1}^{j}(-1)^{l} \tilde{\sigma}_{i_{0} \cdots \hat{i}_{l} \cdots i_{j}}^{j-1}\right] \text { for } j \geqq 1 .
$$

Using the invariance of $\Omega$, the cocycle $f_{i_{0} \cdots i_{4 k-1}}$ can be rewritten as

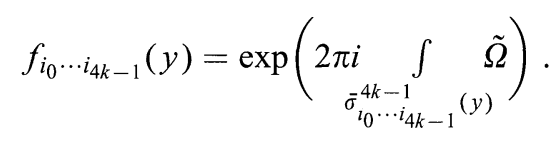

We now lift $f_{i_{0} \cdots i_{4 k-1}}$ to a Čech cocycle with values in the complex of sheaves

$$
\underline{\mathbb{T}}^{d} \stackrel{\log }{\rightarrow} i \cdot \underline{A}_{M}^{1} \rightarrow \cdots \rightarrow i \cdot \underline{A}_{M}^{4 k-1}
$$

where $\underline{A}_{M}^{j}$ is the sheaf of smooth real $j$-forms on $M$. Note that there is the exponential exact sequence $0 \rightarrow 2 \pi i \cdot \mathbb{Z} \rightarrow i \cdot \underline{A}_{M}^{0} \stackrel{\exp }{\rightarrow} \underline{\mathbb{I}} \rightarrow 0$, hence the complex of sheaves (10) is quasi-isomorphic to the complex of sheaves

$$
2 \pi i \cdot \mathbb{Z} \rightarrow i \cdot \underline{A}_{M}^{0} \rightarrow \cdots \rightarrow i \cdot \underline{A}_{M}^{4 k-1},
$$

shifted by one degree to the left. The complex of sheaves (12) is called the smooth Deligne complex and is denoted by $\mathbb{Z}(4 k)_{D}$; it is the smooth analog of the holomorphic Deligne complex [1].

Recall that the Čech hypercohomology of a complex of sheaves $K^{\bullet}$ with respect to $\mathscr{U}=\left(U_{i}\right)$ is the total cohomology of the double complex $C^{p}\left(\mathscr{U}, K^{q}\right)$ of Čech $p$ cochains with coefficients in $K^{q}$, with total differential equal to $\delta+(-1)^{p} d$, where $d$ is the differential of $K^{\bullet}$ and $\delta$ is the Čech differential (see [5]).

The next result gives an explicit Čech cocycle with coefficients in the complex of sheaves $\mathbb{Z}(4 k)_{D}$. We will use the following notations. If we have a product fibration $\Delta^{q} \times U \rightarrow U$ and a smooth mapping $\sigma: \Delta^{q} \times U \rightarrow G$, then for $\alpha$ a $k$ form on $U \times G$ we will denote by $\int_{\sigma} \alpha$ the $(k-q)$-form on $U$ obtained by fiber 
integration of the pull-back of $\alpha$ by the mapping $\left(p_{2}, \sigma\right): \Delta^{q} \times U \rightarrow U \times G$. We extend this construction linearly to any smooth $q$-chain in $\operatorname{Map}(U, G)$.

Theorem 5. (I) For $l \geqq 1$, define an $l$-form $\omega_{i_{0} \cdots i_{4 k-(l+1)}}$ over $U_{i_{0} \ldots i_{4 k-(l+1)}}$ by

$$
\omega_{i_{0} \cdots i_{4 k-(l+1)}}^{l}=(-1)^{\left[\frac{l}{2}\right]} 2^{r(l)} 2 \pi i \int_{\substack{\tilde{\sigma}_{l_{0} \cdots i_{4 k-(l+1)}}^{4 k-(l+1)} \\ i_{0}}} \tilde{\Omega},
$$

where $[m]$ is the greatest integer $\leqq m$, and $r(l)$ is $\left[\frac{l}{2}\right]$, if $l \leqq 2 k$, and $k$ if $l \geqq 2 k$.

Then $\left(f, \omega^{1}, \ldots, \omega^{4 k-1}\right)$ is a Čech $4 k$-cocycle of the covering $\left(U_{l}\right)$, with values in the complex of sheaves $\mathbb{Z}(4 k)_{D}$. Moreover, $d \omega^{4 k-1}=-2^{k-1} \cdot 2 \pi i \alpha$.

(II) The cohomology class of $\left(f, \omega^{1}, \ldots, \omega^{4 k-1}\right)$ in $H^{4 k}\left(M, \mathbb{Z}(4 k)_{D}\right)$ depends only on the principal bundle and the connection over it.

We note that Theorem 5 implies the fact that the Čech cohomology class of $\delta f=2 \pi i \cdot \beta$ corresponds to the de Rham cohomology class of $-2^{k-1} \cdot 2 \pi i \cdot \alpha$, which yields statements (I) and (II) in Theorem 2. Then statement (III) of Theorem 2 will follow because at the last stage of our construction, we have the freedom of adding to each $\sigma_{i_{1} \cdots i_{4 k}}^{4 k-1}$ a smooth singular $(4 k-1)$-cycle in $V_{n, 2 k-1}$, say $\beta_{i_{1} \cdots i_{4 k}}$. The effect of this operation on the Čech cocycle $X_{i_{0} \cdots i_{4 k}}$ is to add to it the coboundary of the Čech cochain $\int_{\beta_{i_{1} \cdots i_{4 k}}} \Omega$; this follows from the definition (3) of $X_{i_{0} \cdots i_{4 k-1}}$ and from the fact that $\Omega$ is an invariant form. Therefore Theorem 2 is a consequence of Theorem 5 .

We thus turn to the proof of Theorem 5. To prove (I), we must show that $d \log s=\delta \omega^{1}$ and that $d \omega^{l}=(-1)^{l} \delta \omega^{l+1}$. Both equations are straightforward applications of the boundary relation (2), and of Stokes' theorem.

We now prove that the cohomology class of $\left(f, \omega^{1}, \ldots, \omega^{4 k-1}\right)$ is independent of the choice of the chains $\sigma^{j}$ and also of the given covering. For the first point, we begin by showing that two homotopic families of $j$-chains $\sigma^{j}$ give the same cohomology class. So, for a given open covering, consider another choice of sections over $U_{i}$ and another choice $\tau_{i_{0} \cdots i_{j}}^{j}$ of smooth chains, satisfying the same assumptions as $\sigma_{i_{0} \cdots i_{j}}^{j}$, and such that there are smooth homotopies $P_{i_{0} \cdots i_{j}}^{j}$ between $\sigma_{i_{0} \cdots i_{j}}^{j}$ and $\tau_{i_{0} \cdots i_{j}}^{j}$, which are compatible with the face relations among these chains. Each $P_{i_{0} \cdots i_{j}}^{j}$ is then a smooth singular cycle in $\operatorname{Map}\left(U_{i_{0} \cdots i_{j}} \times[0,1], V_{n, 2 k-1}\right)$. As before, $\tilde{P}_{i_{0} \cdots i_{j}}^{j}$ will denote the singular cycle in $\operatorname{Map}\left(U_{i_{0} \cdots i_{j}} \times[0,1], S\right)$ obtained from $P_{i_{0} \cdots i_{j}}^{j}$ by means of the trivialization $\bar{s}_{i_{0}}$. We then define a degree Čech $(4 k-1)$-cochain $\left(g, \alpha^{1}, \cdots, \alpha^{4 k-1}\right)$ with values in $\mathbb{Z}(4 k)_{D}$ as follows:

$$
\begin{aligned}
& g_{i_{0} \cdots i_{4 k-2}}=\exp \left(\begin{array}{cc}
2 \pi i \cdot \int_{\tilde{P}_{i_{0}} \cdots i_{4 k-2}} & \int \tilde{\Omega}
\end{array}\right), \\
& \alpha_{i_{0} \cdots i_{4 k-2-j}}^{j}=(-1)^{\left[\frac{j}{2}\right]} 2^{r(j)} 2 \pi i \cdot \int_{\tilde{P}_{i_{0} \cdots i_{4 k-2-j}}}^{\int} \tilde{\Omega} .
\end{aligned}
$$

It is then easy to show, using the boundary relations (2) and Stokes' theorem, that the boundary of this Čech $(4 k-1)$-cochain is the difference between the two cocycles corresponding to the two choices of smooth chains. 
To finish the proof of independence of the choice of the $\sigma^{j}$, we still have to study the effect of modifying each $\sigma_{i_{0} \cdots i_{4 k-1}}^{4 k-1}$ by an integral $(4 k-1)$-cycle; this however has no influence on $f_{i_{0} \cdots i_{4 k-1}}$, as $\Omega$ has integral periods. It is also clear that the construction is invariant under a refinement of the open covering, which finishes the proof of (II).

This concludes the proof of Theorem 5 .

\section{Relation with Differential Characters}

Let $\tilde{p}_{k}$ be the lift of the Pontryagin class constructed in Theorem 5, and denote by $\tilde{e}$ and $\tilde{c}_{k}$ the similar lifts for the Euler and Chern classes. They are classes in the Čech hypercohomology of a smooth Deligne complex of sheaves $\mathbb{Z}(m)_{D}$ (where $m$ is the degree of the characteristic class). Then each of these classes depends on the connection chosen in the construction, so it is natural to compare them to the Chern-Cheeger-Simons Characteristic classes $\hat{p}_{k}, \hat{e}$ and $\hat{c}_{k}$, which take values in the ring of differential characters $[11,12]$. We show in this section that all these classes agree.

To make the comparison, we follow $[15,23]$ and consider the complex

$$
\text { Cone }\left\{A^{\geqq m}(M) \stackrel{\bar{T}}{\rightarrow} S_{s m}^{\bullet}(M ; \mathbb{R} / \mathbb{Z})\right\} .
$$

The notation $A \geqq m(M)$ means truncation from below in the de Rham complex $A^{\bullet}(M), S_{s m}^{\bullet}(M ; \mathbb{R} / \mathbb{Z})$ denotes the complex of smooth singular cochains, and $\bar{T}$ is integration, followed by reduction $\bmod \mathbb{Z}$. The degree $m-1$ cohomology of this complex is exactly $\hat{H}^{m-1}(M)$, the group of degree $m-1$ differential characters, introduced by Cheeger and Simons. The complex (14) is quasi-isomorphic to

$$
\text { Cone }\left\{A^{\geqq m}(M) \rightarrow \operatorname{Cone}\left\{S_{s m}^{\bullet}(M ; \mathbb{Z}) \hookrightarrow S_{s m}^{\bullet}(M ; \mathbb{R})\right\}\right\} .
$$

From a purely algebraic fact about cones, the latter complex identifies with

$$
\text { Cone }\left\{A^{\geqq m}(M) \oplus S_{s m}^{\bullet}(M ; \mathbb{Z}) \rightarrow S_{s m}^{\bullet}(M ; \mathbb{R})\right\} .
$$
to

On the other hand, the Deligne complex of sheaves $\mathbb{Z}(m)_{D}$ is quasi-isomorphic

$$
\text { Cone }\left\{\mathbb{Z} \oplus \underline{A}_{\bar{M}}^{\geqq m} \rightarrow \underline{A}_{M}^{\bullet}\right\}[-1]
$$

where [-1] denotes translation of a complex by one step to the right. The quasiisomorphism in question is given by dividing by $2 \pi i$. The global hypercohomology of the Deligne complex (16) may be computed from a good open covering $\mathscr{U}$ of $M$. If $C^{\bullet}(\mathscr{U},-)$ denotes the complex of Čech cochains of this covering with values in a complex of sheaves, we realize the Deligne cohomology $H^{*}\left(M, \mathbb{Z}(m)_{D}\right)$ as the cohomology of the complex

$$
\text { Cone }\left\{C^{\bullet}(\mathscr{U}, \mathbb{Z}) \oplus C^{\bullet}\left(\mathscr{U}, \underline{A}_{M}^{\geqq m}\right) \rightarrow C^{\bullet}\left(\mathscr{U}, \underline{A}_{M}^{\bullet}\right)\right\}[-1] \text {. }
$$

We denote by $\underline{S}_{s m}^{\bullet}(A)$ the sheaf of smooth singular chains with values in some abelian group $A$. There is a natural map of complexes of sheaves from $\underline{A}_{M}^{\bullet}$ to 
$\underline{S}_{s m}^{\bullet}(\mathbb{R})$, given again by integration. Then there is a natural map of complexes from (17) to the complex

$$
\text { Cone }\left\{C^{\bullet}\left(\mathscr{U}, \underline{S}_{s m}^{\bullet}(\mathbb{Z})\right) \oplus C^{\bullet}\left(\mathscr{U}, \underline{A}_{\bar{M}}^{\geqq m}\right) \rightarrow C^{\bullet}\left(\mathscr{U}, \underline{S}_{s m}^{\bullet}(\mathbb{R})\right)\right\}[-1] .
$$

This is a quasi-isomorphism from (17) to (18).

There is a natural map of complexes from (15) to (18), obtained simply by mapping the global sections of the complex $\operatorname{Cone}\left\{\underline{S}_{s m}^{\bullet}(\mathbb{Z}) \oplus \underline{A}_{\bar{M}}^{\geqq m} \rightarrow \underline{S}_{s m}^{\bullet}(\mathbb{R})\right\}$ to the corresponding Čech double complex. This is again a quasi-isomorphism.

The upshot of this discussion is that both $\hat{H}^{m-1}(M)$ and $H^{m}\left(M ; \mathbb{Z}(m)_{D}\right)$ map isomorphically to the degree $m$ cohomology of (18). All the characteristic classes can then be compared in this complex, and it is enough to show that they agree universally. Narasimhan and Ramanan showed that there is a universal object for principal bundles with connection [21]. In the universal case, all three cohomology groups are isomorphic to the group of closed $m$-forms with integral periods, the isomorphism being induced by exterior differentiation. Since all classes are defined so that their exterior derivative is the appropriate Chern-Weil representative, they must therefore agree. Collecting these results, we have shown:

Proposition 6. The classes $\hat{p}_{k}, \hat{e}, \hat{c}_{k}$ constructed in Theorem 5 agree with the ChernCheeger-Simons classes $\tilde{p}_{k}, \hat{e}$ and $\tilde{c}_{k}$, respectively, in the cohomology of (18).

\section{Special Cases of the Formula}

We will specialize the formula of Theorem 4 to the second Chern class $c_{2}(P)$ of a principal $S U(n)$-bundle $p: P \rightarrow M$. If $\operatorname{dim}(M)=4$, the number $\left\langle c_{2}(P), M\right\rangle$ is called the topological charge or instanton number. There is a canonical choice for an invariant 3-form $\Theta$ on $S U(n)$ generating $H^{3}(S U(n), \mathbb{Z})$, namely the bi-invariant form $\Theta=\frac{1}{24 \pi^{2}} \cdot \operatorname{Tr}\left(g^{-1} d g \wedge g^{-1} d g \wedge g^{-1} d g\right)$. For a point $y \in U_{i j k l m}$, the 3-cycle $Z_{i j k l m}(y)$ is constructed as follows. For each pair of indices $(i, j)$, we choose a path $\tau_{i j}^{1}(y)$ from 1 to $g_{i j}(y)$ in $S U(n)$; we assume that $\tau_{i j}^{1}(y)$ is a smooth function of $y$. It follows from the cocycle condition $g_{i j} g_{j k}=g_{i k}$ that the composition $\tau_{i j}^{1}(y) * g_{i j}(y) \cdot \tau_{j k}^{1}(y) * \tau_{i k}^{1}(y)^{-1}$ is a loop. Since $S U(n)$ is simply-connected, this loop bounds some 2-simplex $\tau_{i j k}^{2}(y)$, which we may take to depend smoothly on $y \in U_{i j k}$. Then we see that the linear combination

$$
g_{i j}(y) \cdot \tau_{j k l}^{2}(y)-\tau_{i k l}^{2}(y)+\tau_{i j l}^{2}(y)-\tau_{i j k}^{2}(y)
$$

is a 2-cycle. Since $S U(n)$ is 2-connected this 2-cycle bounds some 3-simplex $\tau_{i j k l}^{3}(y)$. This may be pictured as a tetrahedron in $S U(n)$ whose vertices are $1, g_{i j}(y), g_{i k}(y), g_{i l}(y)$.

Finally $Z_{i j k l m}(y)$ is defined to be the formal linear combination $-\tau_{i j k l}^{3}(y)+$ $\tau_{i j k m}^{3}(y)-\tau_{i j l m}^{3}(y)+\tau_{i k l m}^{3}(y)-g_{i j} \cdot \tau_{j k l m}^{3}(y)$.

This is a 3 -cycle in $S U(n)$ and our formula for an integral cocycle representing $c_{2}(P)$ becomes

$$
\frac{1}{24 \pi^{2}} \cdot \int_{Z_{i j k l m(y)}} \operatorname{Tr}\left(g^{-1} d g \wedge g^{-1} d g \wedge g^{-1} d g\right)
$$




\section{Remarks}

(i) If we set

$$
f_{i j k l}(y)=\exp \left(2 \pi i \cdot \int_{\tau_{i j k l(y)}^{3}} \frac{1}{24 \pi^{2}} \cdot \operatorname{Tr}\left(g^{-1} d g \wedge g^{-1} d g \wedge g^{-1} d g\right)\right),
$$

then $f$ is a degree 3 Cech cocycle with coefficients in the sheaf $\underline{\mathbb{T}}$. The coboundary of $f_{i j k l}$ in the exponential exact sequence is equal to $2 \pi i$ times our Čech cocycle for $c_{2}$. For $n=2$, the integer associated by our formula to every fivefold intersection of open sets is exhibited as the "winding number" of $f$. This is entirely analogous to the situation which arises with the first Chern class.

(ii) In the case of $S U(2)$, things simplify further since $S U(2)$ is diffeomorphic to $S^{3}$. Then $f_{i j k l}(y)$ can be taken to be the volume of a spherical tetrahedron $\tau_{i j k l}^{3}(y)$. This was found by Laursen, Schierholz and Wiese some years ago [19]. Recall that the formula for the volume of a spherical tetrahedron involves the dilogarithm function.

Next we will describe explicitly the lift $\hat{c}_{2}$ to the smooth Deligne complex of sheaves $\mathbb{Z}(4)_{D}$. This class is represented by a cocycle $\left(f_{i j k l}, \omega_{i j k}^{1}, \omega_{i j}^{2}, \omega_{i}^{3}\right)$. We have here the 0 -cochain $f_{i j k l}$, the 1 -cochain $\omega_{i j k}^{1}$, etc., considered in gauge theory. They are obtained here by specializing the formula in Theorem 5 , and taking into account the simplifications which occur for $\hat{c}_{2}$. To make contact with the gauge theory literature, we denote by $C S(A)$ the Chern-Simons 3-form on $P$ associated to some connection $A$. We introduce simplices $\tilde{\tau}_{i_{0} \cdots i_{j}}^{j}(y)$ in $P$ by setting

$$
\tilde{\tau}_{i_{0} \cdots i_{j}}^{J}(y)=s_{i_{0}}(y) \cdot \tau_{i_{0} \cdots i_{j}}^{j}(y) \text {. }
$$

These are simplices in $P$ whose vertices are the values $s_{i}(y)$ of the local sections; they are fully symmetric with respect to permutations of indices. We define differential forms as follows:

$$
\begin{aligned}
\omega_{i j k}^{1}(\xi)_{y} & =2 \pi i \cdot \int_{\tilde{\tau}_{i j k}^{2}(y)} i_{\tilde{\xi}} \cdot C S(A), \\
\omega_{i j}^{2}\left(\xi_{1}, \xi_{2}\right)_{y} & =-2 \pi i \cdot \int_{\tilde{\tau}_{l J}^{1}(y)} i_{\tilde{\xi}_{1}} \cdot i_{\tilde{\xi}_{2}} \cdot C S(A), \\
\omega_{i}^{3}\left(\xi_{1}, \xi_{2}, \xi_{3}\right)_{y} & =-2 \pi i \cdot C S(A)\left(\tilde{\xi}_{1}, \tilde{\xi}_{2}, \tilde{\xi}_{3}\right)_{s_{i}(y)} .
\end{aligned}
$$

In these formulae, for a vector field $\xi$ over an open set in $M, \tilde{\xi}$ denotes some lift of $\xi$ to $\tilde{\tau}_{i j k}^{2}$, resp. $\tilde{\tau}_{i j}^{1}$, resp. the image of $s_{i}$.

Again, in the case of $S U(2)$, these formulae were essentially known to Laursen, Schierholz and Wiese [19].

(iii) A similar formula for the first Pontryagin class of an $S O(n)$-bundle is given in [6]. The only essential difference lies in the construction of the 3-cycle $X_{i j k l m}(y)$. Again one chooses paths $\sigma_{i j}^{1}(y)$ from 1 to $g_{i j}(y)$, but this time the loop $\sigma_{i j}^{1}(y) *$ $g_{i j}(y) \sigma_{j k}^{1}(y) * \sigma_{i k}^{1}(y)^{-1}$ need not bound. However, since $\pi_{1}(S O(n))=\mathbb{Z}_{2}$ for $n \geqq 3$, twice that loop will bound some 2-simplex. The construction then proceeds as in the case of $c_{2}$, but instead of a 3-simplex one needs a singular 3-chain.

(iv) The case of the first Pontryagin class is very important geometrically. The formulae in that case (and some holomorphic refinements) are used in [7] and in 
[9] to give a geometric construction of a Quillen metric on some determinant line bundles.

(v) Finally, in the case of flat bundles, we recover the formulae of [11, Sect. 8]. The transition functions are then constant and if one chooses the flat connection, the lifts to the smooth Deligne complexes of Proposition 6 reduce to just one component, namely $f_{i_{0} \cdots i}$, which is a Čech cocycle with values in the constant sheaf $\pi$. This component is the exponential of $2 \pi i$ times the volume of some simplex in a Stiefel manifold, and this simplex may be chosen to be totally geodesic. In the case of $\hat{c}_{2}$ we obtain

$$
\exp \left(\frac{i}{12 \pi} \cdot \underset{\tau_{l j k l}^{3}(y)}{\int} \operatorname{Tr}\left(g^{-1} d g \wedge g^{-1} d g \wedge g^{-1} d g\right)\right),
$$

where $\tau_{i j k l}^{3}(y)$ is in fact independent of $y \in U_{i j k l}$.

In the case of the Euler class of a flat real vector bundle, the $(n-1)$-simplex $\gamma_{i_{0}, \ldots, i_{n-1}}^{n-1}(y)$, may be chosen to be a geodesic simplex in $S^{n-1}$, independent of $y$. The formula for the $\mathbb{T}$-valued cocycle becomes

$$
\exp \left(2 \pi i \cdot \operatorname{Vol}\left(\gamma_{i_{0}, \ldots, i_{n-1}}^{n-1}\right)\right)
$$

which is precisely the formula in [11]. The formula of the present paper is more general since it does not require the cocycles $g_{i j}$ to be in general position.

\section{References}

1. Beilinson, A.: Higher regulators and values of L-functions. J. Sov. Math. 30, 2036-2070 (1985)

2. Beresin, F.A., Retakh, V.S.: A method of computing characteristic classes of vector bundles. Rep. Math. Phys. 18, 363-378 (1980)

3. Borel, A.: Sur la cohomologie des espaces fibrés principaux et des espaces homogènes de groupes de Lie compacts. Ann. Math. (2) 57, 115-207 (1953)

4. Borel, A., Hirzebruch, F.: Characteristic classes and homogeneous spaces 2. Am. J. Math. 81, 315-382 (1959)

5. Bott, R., Tu, L.: "Differential forms in algebraic topology". Berlin, Heidelberg, New York: Springer, 1982

6. Brylinski, J.-L., McLaughlin, D.A.: A geometric construction of the first Pontryagin class. In: Quantum Topology, Series on Knots and Everything. L. Kauffman, R. Baadhio, (eds.) Singapore: World Scientific, 1993, pp. 209-220

7. Brylinski, J.-L., McLaughlin, D.A.: Holomorphic quantization and unitary representations of the Teichmüller group. In: Lie Theory and Geometry: In honor of Bertram Kostant, Progress in Math., Birkhaüser, vol. 123, 1994, pp. 21-64

8. Brylinski, J.-L., McLaughlin, D.A.: The geometry of degree 4 characteristic classes and of line bundles on loop spaces I. Duke Math. J. 75, 603-638 (1994)

9. Brylinski, J.-L., McLaughlin, D.A.: The geometry of degree 4 characteristic classes and of line bundles on loop spaces II. Preprint (1995). To appear in Duke Math. J.

10. Cheeger, J.: Spectral geometry of singular riemannian spaces. J. Diff. Geom. 18, 575-657 (1983)

11. Cheeger, J., Simons, J.: Differential characters and geometric invariants. Lecture Notes in Math. vol. 1167, Berlin, Heidelberg, New York: Springer, 1985, pp. 50-80

12. Chern, S.S., Simons, J.: Characteristic forms and geometric invariants. Ann. Math. 99, 48-69 (1974)

13. Dupont, J.: The dilogarithm as a characteristic class for flat bundles. J. Pure Appl. Alg. 44, 137-164 (1987) 
14. Dupont, J.: Characteristic classes for flat bundles and their formulas. Topology 33, 575-590 (1994)

15. Esnault, H.: Characteristic classes of flat bundles. Topology 27, 323-352 (1987)

16. Gabrielov, A., Gel'fand, I.M., Losik, M.V.: Combinatorial calculation of characteristic classes. Funct. Anal. Appl. 9, 48-50, 103-115, 186-202 (1975)

17. Gel'fand, I.M., MacPherson, R.: A combinatorial formula for the Pontryagin classes. Bull. A.M.S. 26, no. 2, 304-309 (1992)

18. Goncharov, A.B.: Explicit construction of characteristic classes. Adv. Soviet Math. 16, Part I 169-210 (1993)

19. Laursen, M.L., Schierholz, G., Wiese, U-J.: 2 and 3-cochains in 4-dimensional $S U(2)$-gauge theory. Commun. Math. Phys. 103, 693-699 (1986)

20. MacPherson, R.: The combinatorial formula of Gabrielov, Gel'fand and Losik for the first Pontryagin class. Séminaire Bourbaki, Exposés 498-506, Lecture Notes in Math. vol. 677, Berlin, Heidelberg, New York, 1977, pp. 105-124

21. Narasimhan, M.S., Ramanan, S.: Existence of universal connections. Am. J. Math. 83, 563-572 (1961) 85, 223-231 (1963)

22. Weil, A.: Sur les théorèmes de de Rham. Commun. Math. Helv. 26, 119-145 (1952)

23. Zucker, S.: The Cheeger-Simons invariant as a Chern class. In: "Algebraic analysis, Geometry and Number Theory, Proc. JAMI Inaugural Conference", JHU Press, 1989, pp. 397-417

Communicated by H. Araki 